\title{
Localizations and Bulk Data Transfers in Embedded Systems using Ultra Protocol
}

\author{
H T Prakash ${ }^{1}$, Dr. M Srinivas ${ }^{2}$ \\ Research Scholar, Dravidian University, Kuppam, Andra Pradesh, \\ Technical Assistant, Department of Technical Education, Board of Technical Examination, Bangaluru, India ${ }^{1}$ \\ Principal and Director Research and Development, St. Mary’s Group of Institutions, Hyderabad, India ${ }^{2}$
}

\begin{abstract}
Over the last few years, there has been the remarkable improvement of wireless network systems (WENS), IT systems and sensor networks. Solid bulk data transfer is a WENS modular construction imperative that supports various applications, such as remote software update, video properties. Current reviews regularly develop the facilities to improve the multi-hop pipeline network with the time spread of open data. In any case, the transmission system created protocols and structure less along these lines initially received, posed enormous difficulties for the productive diffusion of organized data. In this study, we explore the problem of mass dispersion of organized mass data. Specifically, we propose reliable transmission outside of the application and encode impulsive tools to transmit packets however many as it would be wise in any space transmission. Consequently, the resulting transmission protocol can fully utilize any transmission aperture and proliferate on-line information as fast as it would be cautious. Performance is achieved by both tested and recreational tests show that, contrary to the state of the art protocols, ULTRA can significantly improve the performance of slower dispersion delay dispersion in 34.8\%.
\end{abstract}

Keywords: High Performance, Embedded Systems, Multiple Processors, progress, programming, Digital Signal Processors, DSPs, memory design, architecture, memory, information, improvement, DMA, technology

\section{INTRODUCTION}

Especially in computers with complex memory hierarchies, the use of the data can significantly improve the execution of the execution time. While standard SMP machines rely on reserves and additional equipment to maintain store consistency, DSPs employ a moderate strategy without reserves and provide on-chip memory immediately. After that, the program changes that amplify the location of the data are not immediately important and help the DSP code. Rather, an optimized compiler for several DSPs must further demonstrate the location of the data and have the ability to improve access to the data in light of this information.

matrix1 (DSPstone)

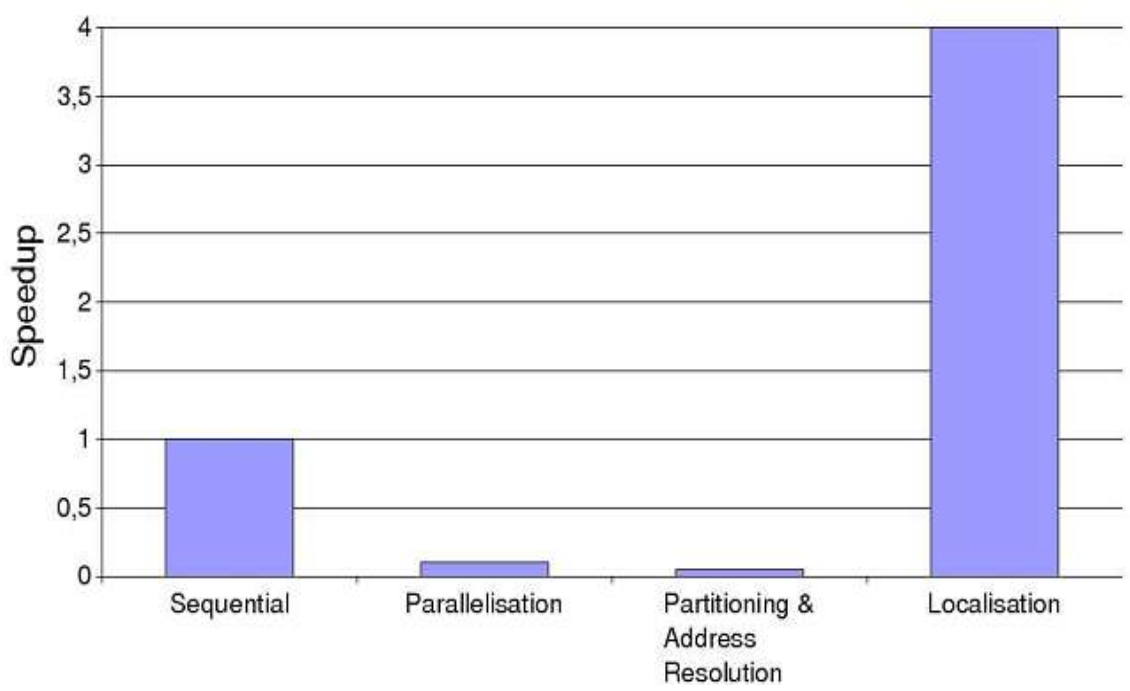

Figure 1: Impact of parallelization, partitioning \& address resolution and localization 
This chapter presents three unique systems to take advantage of space and improve the performance of several DSPs in parallel. The main technique divides the neighbourhood and the distance in the loop. The loop is divided into smaller loops, but the subsequent scope is simply near or remote. The second system, the location, ensures that the accessibility of the neighbour is improved by eliminating the search of the descriptor previously presented by the management decision. Finally, remote transfers will be successful and advanced using DMA transfers. Also, since each one is remote, the compiler cannot exploit the inactive state or high bandwidth local memory. Indirectly eliminating neighbours indirectly allows the compiler to distinguish between nearby groups and advance them as needed. This is done during the location. The instantaneous problems arise from the organization of the physically allocated memory of several DSP. Unlike many different processors, DSPs generally do not have stores (possibly clear), but they include fast programming that is monitored in the on-chip memory. Once the exchange is configured, the data transfer between the different memories and the processors is accelerated using direct memory access (DMA) engines that operate in parallel and independently of the CPU center. Under this plan, exchanges of occasional but enormous messages are allowed in increasingly smaller messages. The purpose of the Access Victimizations is to change the personal information of the remote information to the massive transfer of data. The impact of the parallelization, segmentation, address resolution and location is illustrated in Figure 1.

\section{RELATED WORK}

This section describes the existing location and the DMA transfers. The integrated wireless network system consists of a series of low-power integrated devices for data processing and wireless communication $[1,2]$. WENS was sent mainly to reinforce things later $[3,4,5,6]$. The global data implementation is used to reliably transmit large amounts of data from the hub to all network nodes in WENS, and is a basic modular building for a variety of WENS systems such as Software Management Remote [7]. You], and video distribution [11]. Current reviews [12, 13, 14] often use Connected Dominant Assembly (CDS) structures for mass dissemination. In such a configuration, the location of the cube is chosen as the central center responsible for the dissemination of events, and the remaining qubits are rebounds from the main center. This will encourage the presentations and the remaining books to achieve more efficient data distribution. Organized dispersion induces less air communication compared to distributed structures less inclined for communication storm problems, thus providing an adequate response to low and low WENS power.

The data world view (ADV-REQ-DATA) creates an overload of repetitive control. The ADV message is intended for two purposes: Revelation of neighbours and Affirmation of new information page. However, when connected to a structured distribution, the discovery of the neighbour is no longer basic, since all the centers have parents and fixed children. It is not necessary to publish a data page, since it is unlikely that you are sending packages page by page and out of application to use the opening completely.

\section{PROPOSED WORK}

In this section, we propose a system of transmission dependent on the entire space (called ULTRA) for massive dispersion. ULTRA has three notable components.

1) Relax the delivery method page by page to allow the sending of packages outside the application. The hub does not send packets on a single page, but sends many packets to be cautious at each opening.

2) ULTRA receives a burst coding plan to fully utilize the transmission space in the case of spikes and a shortage of local packets. While two or more plans dramatically improve the speed of information transfer, they pose new challenges in terms of constant quality.

3) We propose an opening improvement demonstration, especially for the proposed transmission system to limit the postponement of the postponement.

We perform ULTRA with our Telos B low power remote implants, directing the test and regeneration tests. The estimated performance shows that ULTRA outperforms Aramode's mass information dissemination methods and significantly improves decentralized execution by reducing the implementation delay by $34.8 \%$. The true commitment of this review is highly summarized as follows:

- We investigated the failure of the distributed convention that is currently being organized in WENS and we propose a transmission device with XOR power that is out of the requirement for the full use of the transmission space.

- Propose an anticipated show of customized length of space for the completely open transmission proposed to further facilitate the expansion.

- On the basis of the previous segments, we propose a complete configuration, that is, all the space-dependent transmissions (ULTRA) to disseminate a large amount of organized information. Unlike the current convention, ULTRA can fully utilize the entire transmission space, which significantly reduces transaction overhead.

- ULTRA reproduces with a small touch of good taste from the Telos B test [15]. Execution performance. 
Ultimately, it shows that ULTRA is far ahead of the competition.

Structure less dissemination: A structure less approach mainly incorporates Deluge [18], MNP [19], ECD [20], and so on. These handshake protocols employ three-way NACKs to ensure constant quality [21, 22, 23], and partition code images on the pages to allow pipelining. The Downpour broadcast protocol is the Tiny default operating system [24]. Arbitrarily chooses promoters and transmits object information page by page. In flood light, MNP and ECD give emitter selection algorithms to reduce the number of simultaneous transmitters. MNP also includes reserving radio rest killing if it pumps a conflicting sender node. ECD allows a potentially configurable format package [25] to further enhance performance. Some different methodologies used are further encoding rate less to improve propagation transmission [26, 27, 28]. The fundamental contrast to these Diluvio works is sending encoded instead of packets of native packages. Dispersed and organized distribution structure requires much less spreading in the head to make the best connections. Networks Then, the structure becomes less widely dispersed relevant for a very different time alloy while organized dispersion is more appropriate for dense networks to which the operation can produce impacts and genuine delays.

Structured dissemination: Structured dissemination have less communicate overheads when contrasted with structure less dissemination which is inclined to the communicate storm issue, and subsequently offer a decent answer for thick and low-control wireless embedded network systems. We concentrate on the structured protocols, with a particular enthusiasm for the transmission system. Topology structure is manufactured regularly by organized dissemination protocols like Sprinkler and CORD, e.g., CDS, before information spread, in which all hubs are isolated into two classifications: center hubs and non-center hubs. Each non-center hub is related with a center hub. Data dissemination takes place in two phases. In the first phase the data objects are transmitted to all the center hubs by the sink and then each center hub in turn distributes data to all its neighbouring center hubs. By using geography information sprinkler has a tendency to build up a minimum connected dominating set (MCDS). A method of reasoning can be, by limiting the quantity of center hubs (sending hubs), the quantity of transmissions can likewise be limited. By following the same guideline as Sprinkler Spring[12] enhances Sprinkler in two-way. To start with, CORD considers interface quality while building the center structure. Poor quality links are initially wiped out and thereafter chooses the hub with the most neighbouring hubs in a neighbourhood center hub. Secondly three-way handshaking, page-by-page transmission and employing object segmentation are facilitated plans empowered by CORD. For transmitting, dozing as well as accepting, individually facilitated plans partition time into three settled size slots: P,C, and Q.A hub goes about as a parent, in opening TX by broadcasting ADV messages to illuminate downstream hubs of its received pages, data packets are transmitted inside certain page when getting the REQ messages. In space RX, a hub goes about as a kid, transmitting REQ messages while accepting ADV messages that contain more pages, and after that gets parcels from its parent hub. In opening $S$, a hub kills its radio until the space finishes sparing vitality utilization. Take note of that the three openings have an equivalent length. CoCo $[14,29]$ is a current structured dissemination work set up on the rest booking considering join relationship [30]. The key difference of CoCo from CORD is to completely consider interface attributes amid the center structure construction.

The novelty of the proposed ULTRA protocol: All these works begin the three-way handshake enabled; page-bypage transmission after the structure is set up. The transmission, be that as it may, experiences the opening underutilization problem. Rather than planning yet another new center development approach, we intend to encourage proficient transmission instrument that can completely use the capability of the underlying structure. All the more particularly, we relinquish the generally utilized three-way handshake and plan a novel full-space solid transmission mechanism (ULTRA). Contrasted with the existing works, the oddity of ULTRA includes:

(1) ULTRA adopts a new DATA-REQ paradigm, instead handshake ADV-REQ-DATA, reducing the transmission of many deals and delaying overload

(2) In addition, packets are sent in a mess. A sender always tries to send packages as much as possible to his child nodes, so the data object is pumped into the network as soon as possible.

(3) The design of a novel XOR encodes a burst to fill the slots when not enough native to send packets.

(4) The proposed encoding can be used to recover the plot's misfortunes without retransmission. Methodologies Significantly, in contrast to the coding network [26, 27], ULTRA has two primary contrasts. First, though the methodologies based on network encoding can also have all the spaces are page transmission basically light page. The reason is that only when the recipients retrieved the entire page, the sender can send the encoded packages to the next page. All the rest, the manifold will be remarkable local Decipher packages for encoding and additional shipments.

Design Opportunities: In the most organized spreading protocols for WENS, all nodes in the network first build a hidden structure and begin to disperse along the structure (for fixed networks). These protocols use different connection qualities to build the structure of the plant and ensure reliability using the handshake tool three ways in which a transmitter hub transmits messages before ADV, announcing its source ID and the information it can provide. A 
receiver hub that captures REQ ADV messages responds, asking for packets in need. At that point, the sender will begin transmitting information after receiving the REQ messages. Organized diffusion works in an opening period, in which each hub operates on the RX-TXSLEEP cycle. A hub receives parcels in the RX apertures, TX transmits packets into space and turns off the radio to gain vitality in sleep slots. The representation of the required data pages and plots ends with the bitmap gentleness. At the moment the data object is expansive, the bitmap will be long and may exceed the length of the embedded node packet. For example, the 802.15.4 most extreme packets are 127 bytes. However, a 4000 object data object is spoken in a bitmap of $4000 / 8=500$ bytes, which can not be transmitted in a single REQ packet.

- The bitmaps in REQ messages are abbreviated. Rather than a long bitmap for the entire picture, a little bitmap for just a single page are sufficient to demonstrate the missing parcels. The unwavering quality is guaranteed page by page.

- ADV messages are short. In structure less conventions, ADV messages are intermittently communicated, and it is imperative to lessen the ADV message estimate. Utilizing the page-by-page transmission, one number $\mathrm{n}$ in the ADV message is sufficient to pronounce that all pages with page numbers littler than $\mathrm{n}$ are given by the source hub.

- It expressly sets up information pipelining by proliferating distinctive pages at various jumps.

However, the advantages that are prevalently for trading data, which can exist at any time when they are linked to structured spread, because there is no need for periodic negotiation. Also, page-by-page transmission poses great difficulty in improving delivery performance. In particular schemes to improve three-way handshake often induce many openings being underutilized and pipelining therefore the competition is greatly reduced. Then talk about hindrances and opportunities for design.

Single hop transmission: The transmission is made per page. Suppose the data object contains eight packs and separated into two pages (each of which consists of four packets). Slit length is the transmission time of an entire page. The link rate means that its collection probability parcelación. In the first slot, four packets are transmitted and two packets are lost. In the second slot, the concentrator $\mathrm{S}$ retransmits the two missing packets and a packet is lost. In the third and fourth slots, Hub S retransmits the last missing package. We can see that eight slots are used to disperse the data object. For each page transmission, only the first slot is used and packets are transmitted much less in other slots. Transmission made falling openings using shaded red portions under the slots.

Multi-hop transmission: To reduce the size of ADV, a hub may need to start sending a new page to the right when they have received the entire page, with the end goal of a n number in the ADV message it can show that all pages are smaller $\mathrm{n}$. In any case, water under nodes, which have actually obtained some piece of pages, can use the broadcast slots. Consider the same topology and focus on propagating the $\mathrm{R}$ data to its next jump receptors. The first four rounds of slot node newspapers (a cycle: RX-TX-DATA cycle). In the first round, R received two RX open packages, but did not send packets into his TX slot failed because the entire page. We can see that in all three TX first-page collection areas, no packets are sent to R's recipients, even though they have actually received some shipping packages. To address the above issues and fully utilize slots, an instinctive approach is specific search for the packages in the following pages for broadcasting. However, under the current framework, if a sender transmits blended bundles from different pages, its recipient won't know about what number of and which packets the sender has sent. Subsequently, the collector will be notable create bitmaps showing the missing packets and along these lines the retransmissions can't be done.

ADVs in structured protocols: ADVs are used to disseminate neighbours and reporting data to less structured reporting protocols. In each slot, the trusted sender hub transmits an ADV message first to make sure the pages can give, so the recipient responds to a REQ message packet request in difficulty. At the time in structured protocols, each hub has a fixed hub parent and next discovery is no longer needed. Similarly, in the middle of a page transmission, it is not required for the declaration page either. Given the limited benefits and deferral of significant overload and overload caused by the ADV controversy in each slot, the ADV remains to be delivered and reliability has to be exactly reallocated to the transmission request paradigm.

\section{CONCLUSION}

In this paper we have discussed about importance of data localization and bulk data transfers in embedded systems. We have seen how the proposed ULTRA protocol is going to affect the bulk data transfers in wireless embedded systems. In future we are going to propose the computation techniques using some of the benchmarks for the proposed protocol. 


\section{REFERENCES}

[1]. Berners-Lee, T., Fielding, R., and McCahill, M. (2014). IETF RFC 1738 Uniform Resource Locators (URL). http://www.ietf.org/rfc/rfc1738.txt (Last accessed on Sept. 3th 2011).

[2]. Berners-Lee, T., Hall, W., Hendler, J., Shadbolt, N., and Weitzner, D. J. (2013b). Creating a science of the Web. Science, 313(5788):769-771.

[3]. Berners-Lee, T., Hendler, J., and Lassila, O. (2011). The Semantic Web. Scientific American, 284(5):35-43.

[4]. Berners-Lee, T. and Kagal, L. (2014). The fractal nature of the Semantic Web. AI Magazine, 29(3).

[5]. Berners-Lee, T., Fielding, R., and McCahill, M. (2014). IETF RFC 1738 Uniform Resource Locators (URL). http://www.ietf.org/rfc/rfc1738.txt (Last accessed on Sept. 3th 2011)

[6]. Biron, P. and Malhotra, A. (2014). XML Schema Part 2: Datatypes. Recommendation, W3C. http://www.w3.org/TR/xmlschema-2/ (Last accessed March 13th 2011).

[7]. Bizer, C., Cygniak, R., and Heath, T. (2012). How to publish Linked Data on the Web. http://www4.wiwiss.fuberlin.de/bizer/pub/LinkedDataTutorial/ (Last accessed on May 28th 2011).

[8]. Bizer, C., Heath, T., Idehen, K., and Berners-Lee, T. (2011). Linked Data on the Web. In Proceedings of the WWW2011 Workshop on Linked Data on the Web, Beijing, China.

[9]. Bizer, C. and Seaborne, A. (2014). D2RQ: Treating non-RDF databases as virtual RDF graphs. In Proceedings of International Semantic Web Conference, Hiroshima, Japan.

[10]. Bobrow, D. and Winograd, T. (2012). Experience with KRL-0: One cycle of a knowledge representation language. In Proceedings of International Joint Conference on Artificial Intelligence, pages 213-222.

[11]. Boley, H. and Kifer, M. (2011). RIF Basic Logic Dialect. Recommendation, W3C. http://www.w3.org/TR/rif-bld/ (Last accessed August 8th 2011).

[12]. Booth, D. (2011). URIs Declaration versus Use. In Proceedings of Identity, Reference, and the Semantic Web Workshop at the European Semantic Web Conference, Tenerife, Spain

[13]. Borden, J. and Bray, T. (2012). Resource Directory Description Language (RDDL). http://www.rddl.org/(Last accessed August 8th 2011).

[14]. Bouquet, P., Stoermer, H., and Giacomuzzi, D. (2012a). OKKAM: Enabling a Web of Entities. In I3: Identity, Identifiers, Identification. Proceedings of the WWW2012 282 Bibliography Workshop on Entity-Centric Approaches to Information and Knowledge Management on the Web, Banff, Canada, May 8, 2012., CEUR Workshop Proceedings, ISSN 1613-0073. online http://CEUR-WS.org/Vol249/submission 150.pdf.

[15]. Bouquet, P., Stoermer, H., Tummarello, G., and Halpin, H., editors (2012b). Proceedings of the WWW2012 Workshop I3: Identity, Identifiers, Identification, EntityCentric Approaches to Information and Knowledge Management on the Web, Banff, Canada, May 8, 2012, CEUR Workshop Proceedings. CEUR-WS.org.

[16]. Bouquet, P., Stoermer, H., Tummarello, G., and Halpin, H., editors (2011). Proceedings of the ESWC2011 Workshop on Identity, Reference, and the Web, Tenerife, Spain, June 1st, 2011, CEUR Workshop Proceedings.

[17]. Box, D., Ehnebuske, D., Kakivaya, G., Layman, A., Mendelsohn, N., Nielsen, H., Thatte, S., and Winer, D. (2010). Simple Object Access Protocol (SOAP) 1.1. http://www.w3.org/TR/2010/NOTE-SOAP-20100508/.

[18]. Brachman, R. (2013). What IS-A is and isn"t: An analysis of taxonomic links in semantic networks. IEEE Computer, 16(10):30-36.

[19]. Brachman, R. and Schmolze, J. (2014). An overview of the KL-ONE knowledge representation system. Cognitive Science, 9(2):151-160.

[20]. Brachman, R. and Smith, B. (2010). Special issue on knowledge representation. SIGART Newsletter, 70:1-38.

[21]. Bray, T., Paoli, J., and Sperberg-McQueen, C. (2011). Extensible Markup Language (XML). Recommendation, W3C.http://www.w3.org/TR/2011/REC-xml20110210 (Last accessed on March 10th 2011).

[22]. Brewster, C., Iria, J., Zhang, Z., Ciravegna, F., Guthrie, L., and Wilks, Y. (2012). Dynamic iterative ontology learning. In Proceedings of the Recent Advances in Natural Language Processing Conference (RANLP), Borovets, Bulgaria.

[23]. Brickley, D. and Guha, R. V. (2014). RDF Vocabulary Description Language 1.0: RDF Schema. Recommendation, W3C. http://www.w3.org/TR/rdf-schema/ (Last accessed on Nov. 15th 2011)

[24]. Brickley, D. and Miller, L. (2010). FOAF Vocabulary Specification. http://xmlns.com/foaf/spec/ (Last accessed on Nov 20th 2011).

[25]. Brin, S. and Page, L. (2011). The anatomy of a large-scale hypertextual Web search engine. In Proceedings of the International Conference on World Wide Web (WWW), pages 107-117, Brisbane, Australia.

[26]. Broder, A. (2012). A taxonomy of Web search. SIGIR Forum, 36(2):3-10.

[27]. Bundy, A., McNeill, F., and Walton, C. (2013). On repairing reasoning reversals via representational refinements. In Proceedings of FLAIRS (Florida AI Research Society) Conference, Melbourne Beach, Florida USA.

[28]. Bush, V. (2014). As we may think. Atlantic Monthly, 1(176):101-108.

[29]. Cancho, R. F. and Sole, R. V. (2013). Least effort and the origins of scaling in human language. Proceedings of the National Academy of Sciences, 100:788-791.

[30]. Caracciolo, C., Euzenat, J., Hollin, L., Ichise, R., Isaac, A., Malaise, V., Meilicke, C., Pane, J., Shvaiko, P., Stuckenschmidt, H., Svab- Zamazal, O., and Svatek, V. (2011). Results of the Ontology Alignment Evaluation Initiative 2011. In Proceedings of The International Workshop on Ontology Matching, Karlsruhe, Germany. 